\title{
Borel superrigidity and the classification problem for the torsion-free abelian groups of finite rank
}

\author{
Simon Thomas*
}

\begin{abstract}
In 1937, Baer solved the classification problem for the torsion-free abelian groups of rank 1. Since then, despite the efforts of many mathematicians, no satisfactory solution has been found of the classification problem for the torsion-free abelian groups of rank $n \geq 2$. So it is natural to ask whether the classification problem for the higher rank groups is genuinely difficult. In this article, I will explain how this question can be partially answered, using ideas from descriptive set theory and Zimmer's superrigidity theory.
\end{abstract}

Mathematics Subject Classification (2000). Primary 03E15, 20K15, 37A20.

Keywords. Borel equivalence relation, superrigidity, torsion-free abelian group.

\section{Introduction}

In this article, we shall discuss some recent work which partially explains why no satisfactory system of complete invariants has yet been found for the torsion-free abelian groups of finite rank $n \geq 2$. Recall that, up to isomorphism, the torsion-free abelian groups $A$ of rank $n$ are exactly the additive subgroups of the $n$-dimensional vector space $\mathbb{Q}^{n}$ which contain $n$ linearly independent elements. Thus the classification problem for the torsion-free abelian groups of rank $n$ can be naturally identified with the corresponding problem for

$$
R\left(\mathbb{Q}^{n}\right)=\left\{A \leqslant \mathbb{Q}^{n} \mid A \text { contains } n \text { linearly independent elements }\right\} .
$$

In 1937, Baer [3] solved the classification problem for the class $R(\mathbb{Q})$ of torsionfree abelian groups of rank 1 as follows. Let $\mathbb{P}$ be the set of primes. Suppose that $G \in R(\mathbb{Q})$ and that $0 \neq x \in G$. Then for each $p \in \mathbb{P}$, the $p$-height of $x$ is defined to be

$$
h_{x}(p)=\sup \left\{n \in \mathbb{N} \mid \text { There exists } y \in G \text { such that } p^{n} y=x\right\} \in \mathbb{N} \cup\{\infty\} ;
$$

and the characteristic $\chi(x)$ of $x$ is defined to be the sequence

$$
\left\langle h_{x}(p) \mid p \in \mathbb{P}\right\rangle \in(\mathbb{N} \cup\{\infty\})^{\mathbb{P}} .
$$

\footnotetext{
* Research partially supported by NSF Grants.
} 
Two sequences $\chi_{1}, \chi_{2} \in(\mathbb{N} \cup\{\infty\})^{\mathbb{P}}$ are said to belong to the same type, written $\chi_{1} \equiv \chi_{2}$, iff

(a) $\chi_{1}(p)=\chi_{2}(p)$ for almost all primes $p$; and

(b) if $\chi_{1}(p) \neq \chi_{2}(p)$, then both $\chi_{1}(p)$ and $\chi_{2}(p)$ are finite.

Clearly $\equiv$ is an equivalence relation on $(\mathbb{N} \cup\{\infty\})^{\mathbb{P}}$. Furthermore, it is easily checked that if $G \in R(\mathbb{Q})$, then $\chi(x) \equiv \chi(y)$ for all $0 \neq x, y \in G$. Hence we can define the type $\tau(G)$ of $G$ to be the $\equiv$-equivalence class containing $\chi(x)$, where $x$ is any non-zero element of $G$. In [3], Baer proved that $\tau(G)$ is a complete invariant for the isomorphism problem for the rank 1 groups.

Theorem 1.1 (Baer [3]). If $G, H \in R(\mathbb{Q})$, then $G \cong H$ iff $\tau(G)=\tau(H)$.

However, the situation is much less satisfactory in the case of the torsion-free abelian groups of rank $n \geq 2$. In the late 1930s, Kurosh [22] and Malcev [25] found complete invariants for these groups consisting of equivalence classes of infinite sequences $\left\langle M_{p} \mid p \in \mathbb{P}\right\rangle$ of matrices, where each $M_{p} \in \mathrm{GL}_{n}\left(\mathbb{Q}_{p}\right)$. Unfortunately, as Fuchs [8] remarks in his classic textbook, the associated equivalence relation is so complicated that the problem of deciding whether two sequences are equivalent is as difficult as that of deciding whether the corresponding groups are isomorphic. It is natural to ask whether the classification problem for the higher rank groups is genuinely more difficult than that for the rank 1 groups. Of course, if we wish to show that the classification problem for the groups of rank $n \geq 2$ is intractible, it is not enough merely to prove that there are $2^{\aleph_{0}}$ such groups up to isomorphism: for there are $2^{\aleph_{0}}$ pairwise nonisomorphic groups of rank 1 and we have just seen that Baer has given a satisfactory classification for this class of groups. In this article, following Friedman-Stanley [7] and Hjorth-Kechris [14], we shall explain how to use the more sensitive notions of descriptive set theory to measure the complexity of the classification problem for the groups of rank $n \geq 2$.

The basic idea is quite simple; namely, in order to understand the relative complexity of these and other classification problems, we shall consider the question of when one classification problem can be "explicitly reduced" to another. For example, the classification problem for the rank $n$ groups can be explicitly reduced to that for the rank $n+1$ groups by the map

$$
\begin{aligned}
R\left(\mathbb{Q}^{n}\right) & \rightarrow R\left(\mathbb{Q}^{n+1}\right) \\
A & \mapsto A \oplus \mathbb{Q}
\end{aligned}
$$

in the sense that

$$
A \cong B \quad \text { iff } \quad A \oplus \mathbb{Q} \cong B \oplus \mathbb{Q} .
$$

Of course, this observation is neither surprising nor particularly interesting; and we shall be more concerned with the question of whether there exists an "explicit map" in the opposite direction

$$
f: R\left(\mathbb{Q}^{n+1}\right) \rightarrow R\left(\mathbb{Q}^{n}\right)
$$


such that

$$
A \cong B \quad \text { iff } \quad f(A) \cong f(B) .
$$

If we drop the requirement that $f$ should be "explicit", then such a map certainly exists: since $R\left(\mathbb{Q}^{n+1}\right)$ and $R\left(\mathbb{Q}^{n}\right)$ both contain $2^{\aleph_{0}}$ groups up to isomorphism, we can simply use the Axiom of Choice to match up the isomorphism classes. However, nobody would regard such a matching as a satisfactory reduction of one classification problem to another. In order to give a precise formulation of this question, it is first necessary to discuss some of the basic notions from the theory of Borel equivalence relations.

Let $(X, \diamond)$ be a measurable space; i.e. a set $X$ equipped with a $\sigma$-algebra $\delta$ of subsets of $X$. Then $(X, \wp)$ is said to be a standard Borel space iff there exists a complete separable metric $d$ on $X$ such that $\delta$ is the $\sigma$-algebra of Borel sets of $(X, d)$. By a classic result of Kuratowski [21], if $(X, \S)$ is an uncountable standard Borel space, then $(X, \delta)$ is measurably isomorphic to the unit interval $[0,1]$ equipped with its $\sigma$-algebra of Borel sets. The obvious examples of standard Borel spaces include $\mathbb{R}, \mathbb{C}$ and $\mathbb{Q}_{p}$, as well as the Cantor space

$$
2^{C}=\{h \mid h: C \rightarrow 2\},
$$

where $C$ is any countably infinite set. Furthermore, identifying each subset $B \subseteq C$ with its characteristic function $\chi_{B} \in 2^{C}$, it follows that the power set $\mathcal{P}(C)$ is also a standard Borel space. Less obviously, there is a uniform way to represent classes of countable structures, such as groups, fields, graphs, etc., by the elements of suitable standard Borel spaces. For example, in order to define the standard Borel space of countable graphs, we first restrict our attention to the set $\mathcal{C}$ of graphs

$$
\Gamma=\left\langle\mathbb{N}, E_{\Gamma}\right\rangle
$$

with vertex set $\mathbb{N}$. After identifying each such graph $\Gamma \in \mathcal{C}$ with its edge relation $E_{\Gamma} \in \mathcal{P}(\mathbb{N} \times \mathbb{N})$, it is easily checked that $\mathcal{C}$ is a Borel subset of the standard Borel space $\mathcal{P}(\mathbb{N} \times \mathbb{N})$; and this implies that $\mathcal{C}$ is also a standard Borel space. (For example, see Kechris [20].) It should be relatively clear how to generalise the method of this example to deal with other classes of countable structures. However, in this article, we shall mainly be concerned with the classes of torsion-free abelian groups of rank $n \geq 1$ and the class of finitely generated groups; and these classes can be more conveniently represented by the following more ad hoc spaces.

Example 1.2. Let $n \geq 1$. Then $R\left(\mathbb{Q}^{n}\right)$ is a Borel subset of the standard Borel space $\mathcal{P}\left(\mathbb{Q}^{n}\right)$ and so $R\left(\mathbb{Q}^{n}\right)$ is a standard Borel space. For later use, note that the natural action of $\mathrm{GL}_{n}(\mathbb{Q})$ on the vector space $\mathbb{Q}^{n}$ induces a corresponding action on $R\left(\mathbb{Q}^{n}\right)$; and that if $A, B \in R\left(\mathbb{Q}^{n}\right)$, then $A \cong B$ iff there exists an element $\varphi \in \mathrm{GL}_{n}(\mathbb{Q})$ such that $\varphi[A]=B$. 
Example 1.3 (Champetier [4]). The standard Borel space $g$ of finitely generated groups can be defined as follows. Let $F$ be the free group on countably many generators $X=\left\{x_{i} \mid i \in \mathbb{N}\right\}$. Suppose that $G$ is a finitely generated group and that $\left(g_{0}, \ldots, g_{n}\right)$ is a finite sequence of generators. Then, by considering the homomorphism $\pi: F \rightarrow G$ defined by

$$
\pi\left(x_{i}\right)= \begin{cases}g_{i} & \text { if } 0 \leq i \leq n, \\ 1 & \text { otherwise }\end{cases}
$$

we see that $G$ can be realized as a quotient $F / N$, where $N$ is a normal subgroup which contains all but finitely many elements of the basis $X$. (Of course, choosing a different generating sequence usually results in a different realization.) Thus we can identify $g$ with the set of all such normal subgroups $N$ of $F$. With this identification, $g$ is a Borel subset of the standard Borel space $\mathcal{P}(F)$ and hence $g$ is a standard Borel space. As in Example 1.2, the isomorphism relation on the standard Borel space of finitely generated groups is the orbit equivalence relation of a natural action of a suitable countable group. More precisely, let $\operatorname{Aut}_{f}(F)$ be the subgroup of $\operatorname{Aut}(F)$ generated by the elementary Nielsen transformations

$$
\left\{\alpha_{i} \mid i \in \mathbb{N}\right\} \cup\left\{\beta_{i j} \mid i \neq j \in \mathbb{N}\right\},
$$

where $\alpha_{i}$ is the automorphism sending $x_{i}$ to $x_{i}^{-1}$ and leaving $X \backslash\left\{x_{i}\right\}$ fixed; and $\beta_{i j}$ is the automorphism sending $x_{i}$ to $x_{i} x_{j}$ and leaving $X \backslash\left\{x_{i}\right\}$ fixed. Then the natural action of $\operatorname{Aut}_{f}(F)$ on $F$ induces a corresponding action on the space $g$ of normal subgroups of $F$ which contain all but finitely many elements of the basis $X$; and if $N, M \in g$ are two such normal subgroups, then $F / N \cong F / M$ iff there exists $\varphi \in \operatorname{Aut}_{f}(F)$ such that $\varphi[N]=M$. (For example, see Champetier [4] and Lyndon-Schupp [24].)

If $X, Y$ are standard Borel spaces, then $f: X \rightarrow Y$ is a Borel map iff $f^{-1}(B)$ is Borel for every Borel subset $B \subseteq Y$. Equivalently, $f$ is Borel iff graph $(f)$ is a Borel subset of $X \times Y$. Now suppose that $E, F$ are equivalence relations on the standard Borel spaces $X, Y$ respectively. (For example, $X$ and $Y$ could be spaces of countable structures and $E, F$ could be the corresponding isomorphism relations.) Then $E$ is Borel reducible to $F$, written $E \leq_{B} F$, if there exists a Borel map $f: X \rightarrow Y$ such that

$$
x E y \quad \text { iff } \quad f(x) F f(y) .
$$

$E$ and $F$ are Borel bireducible, written $E \sim_{B} F$, if both $E \leq_{B} F$ and $F \leq_{B} E$. Finally we write $E<_{B} F$ if both $E \leq_{B} F$ and $F ⿱_{B} E$.

Remark 1.4. Of course, the notion of a Borel reduction $f: X \rightarrow Y$ from $E$ to $F$ is intended to capture the intuitive idea of an "explicit reduction" from the $E$-classification problem to the $F$-classification problem. For example, with a little practice, it is easily checked that any given explicit map $f: \mathbb{R} \rightarrow \mathbb{R}$ is Borel. On the other hand, many mathematicians are reluctant to accept that an arbitrary Borel map $f: \mathbb{R} \rightarrow \mathbb{R}$ should 
be regarded as explicit. However, it is not necessary for us to address this question, since we will mainly be concerned with non-reducibility results; and for such results, it is clearly preferable to work with the broadest possible class of maps. (It is perhaps worth mentioning that the proofs of our main results actually show that there are no measurable reductions between the relevant classification problems. By a well-known theorem of Solovay [30], the existence of a non-measurable map requires an essential use of the Axiom of Choice and so such maps are certainly not explicit.)

Example 1.5. For each $n \geq 1$, let $\cong_{n}$ be the isomorphism relation on $R\left(\mathbb{Q}^{n}\right)$. Then the map

$$
\begin{aligned}
R\left(\mathbb{Q}^{n}\right) & \rightarrow R\left(\mathbb{Q}^{n+1}\right), \\
A & \mapsto A \oplus \mathbb{Q},
\end{aligned}
$$

is a Borel reduction from $\cong_{n}$ to $\cong_{n+1}$. Hence

$$
\left(\cong_{1}\right) \leq_{B}\left(\cong_{2}\right) \leq_{B} \cdots \leq_{B}\left(\cong_{n}\right) \leq_{B} \cdots
$$

and our earlier question of whether the classification problem for the higher rank groups is genuinely more difficult than that for the rank 1 groups can be interpreted as the question of whether $\left(\cong_{1}\right)<_{B}\left(\cong_{2}\right)$.

Before discussing the solution of this problem, it will be helpful to give a brief account of some of the theory of countable Borel equivalence relations. (A detailed development of this theory can be found in Jackson-Kechris-Louveau [17].) If $X$ is a standard Borel space, then a Borel equivalence relation on $X$ is an equivalence relation $E \subseteq X^{2}$ which is a Borel subset of $X^{2}$. The Borel equivalence relation $E$ is said to be countable iff every $E$-equivalence class is countable. Most of the Borel equivalence relations that we shall consider in this article arise from group actions as follows. Let $G$ be an $l c s c$ group; i.e. a locally compact second countable group. Then a standard Borel $G$-space is a standard Borel space $X$ equipped with a Borel action $(g, x) \mapsto g \cdot x$ of $G$ on $X$. The corresponding $G$-orbit equivalence relation on $X$, which we shall denote by $E_{G}^{X}$, is a Borel equivalence relation. In fact, by Kechris [19], $E_{G}^{X}$ is Borel bireducible with a countable Borel equivalence relation. Conversely, by Feldman-Moore [6], if $E$ is an arbitrary countable Borel equivalence relation on the standard Borel space $X$, then there exists a countable group $G$ and a Borel action of $G$ on $X$ such that $E=E_{G}^{X}$.

Example 1.6. As we pointed out in Examples 1.2 and 1.3, the isomorphism relations on the spaces $R\left(\mathbb{Q}^{n}\right)$ of torsion-free abelian groups of rank $n$ and the space $g$ of finitely generated groups are the orbit equivalence relations of natural actions of suitable countable groups. These actions are easily seen to be Borel and so each of these isomorphism relations is a countable Borel equivalence relation.

With respect to Borel reducibility, the least complex countable Borel equivalence relations are those which are smooth; i.e. those countable Borel equivalence relations $E$ on a standard Borel space $X$ for which there exists a Borel function $f: X \rightarrow Y$ 
into a standard Borel space $Y$ such that $x E y$ iff $f(x)=f(y)$. Equivalently, the countable Borel equivalence relation $E$ on $X$ is smooth iff the quotient $X / E$ is a standard Borel space. (Here $X / E$ denotes the set of $E$-classes equipped with the quotient Borel structure.)

Example 1.7. The isomorphism relation on the standard Borel space of countable divisible abelian groups is smooth. To see this, recall that if $A$ is a countable divisible abelian group, then $A=D \oplus T$, where $T$ is the torsion subgroup and $D$ is torsion-free. Let $r_{0}(A) \in \mathbb{N} \cup\{\infty\}$ be the rank of $D$; and for each prime $p$, let $r_{p}(A) \in \mathbb{N} \cup\{\infty\}$ be the rank of the $p$-component $T_{p}$ of $T$. Then the invariant

$$
\rho(A)=\left(r_{0}(A), r_{2}(A), r_{3}(A), \ldots, r_{p}(A), \ldots\right)
$$

determines $A$ up to isomorphism.

Next in complexity come those countable Borel equivalence relations $E$ which are Borel bireducible with the Vitali equivalence relation $E_{0}$ defined on $2^{\mathbb{N}}$ by $x E_{0} y$ iff $x(n)=y(n)$ for almost all $n$. More precisely, by Harrington-Kechris-Louveau [12], if $E$ is a countable Borel equivalence relation, then $E$ is nonsmooth iff $E_{0} \leq_{B} E$. Furthermore, by Dougherty-Jackson-Kechris [5], if $E$ is a countable Borel equivalence relation on a standard Borel space $X$, then the following three properties are equivalent:

(1) $E \leq{ }_{B} E_{0}$.

(2) $E$ is hyperfinite; i.e. there exists an increasing sequence

$$
F_{0} \subseteq F_{1} \subseteq \cdots \subseteq F_{n} \subseteq \cdots
$$

of finite Borel equivalence relations on $X$ such that $E=\bigcup_{n \in \mathbb{N}} F_{n}$. (Here an equivalence relation $F$ is said to be finite iff every $F$-equivalence class is finite.)

(3) There exists a Borel action of $\mathbb{Z}$ on $X$ such that $E=E_{\mathbb{Z}}^{X}$.

Example 1.8. As is easily checked, Baer's classification of the rank 1 groups implies that $\left(\cong_{1}\right) \sim_{B} E_{0}$.

It turns out that there is also a most complex countable Borel equivalence relation $E_{\infty}$, which is universal in the sense that $F \leq_{B} E_{\infty}$ for every countable Borel equivalence relation $F$; and, furthermore, $E_{0}<_{B} E_{\infty}$. (Clearly this universality property uniquely determines $E_{\infty}$ up to Borel bireducibility.) $E_{\infty}$ has a number of natural realisations in many areas of mathematics, including algebra, topology and recursion theory. (See Jackson-Kechris-Louveau [17].) For example, $E_{\infty}$ is Borel bireducible with both the isomorphism relation for finitely generated groups [36] and the isomorphism relation for fields of finite transcendence degree [37].

For many years, it was an open problem whether there existed infinitely many countable Borel equivalence relations $E$ such that $E_{0}<_{B} E<_{B} E_{\infty}$. This problem 
was finally resolved by Adams-Kechris [2], who used Zimmer's superrigidity theory [38] to show that there are actually $2^{\aleph_{0}}$ such relations $E$ up to Borel bireducibility. More recently, Hjorth-Kechris [15] have found an "elementary" proof of this result; i.e. a proof which requires no more background than the standard measure theory and functional analysis which should be known by every mathematician.

Returning to our discussion of the complexity of the isomorphism relation $\cong_{n}$ on the standard Borel space $R\left(\mathbb{Q}^{n}\right)$ of torsion-free abelian groups of rank $n$, we now see that

$$
\left(\cong_{1}\right) \leq_{B}\left(\cong_{2}\right) \leq_{B} \cdots \leq_{B}\left(\cong_{n}\right) \leq_{B} \cdots \leq_{B} E_{\infty} .
$$

In [14], Hjorth-Kechris conjectured that $\left(\cong_{2}\right) \sim_{B} E_{\infty}$; in other words, the classification problem for the torsion-free abelian groups of rank 2 is already as complex as that for arbitrary finitely generated groups. Of course, if true, this would have completely explained the failure to find a satisfactory system of complete invariants for the torsion-free abelian groups of rank $n \geq 2$, since nobody expects such a system to exist for the class of finitely generated groups. In [13], Hjorth provided some initial evidence for this conjecture by proving that the classification problem for the higher rank groups is indeed genuinely more difficult than that for the rank 1 groups.

Theorem 1.9 (Hjorth [13]). $\left(\cong_{1}\right)<_{B}\left(\cong_{2}\right)$.

However, the conjecture appeared considerably less plausible after Adams-Kechris [2] used Zimmer's superrigidity theory [38] to prove that

$$
\left(\cong_{1}^{*}\right)<_{B}\left(\cong_{2}^{*}\right)<_{B} \cdots<_{B}\left(\cong_{n}^{*}\right)<_{B} \cdots
$$

where $\left(\cong_{n}^{*}\right)$ is the restriction of the isomorphism relation to the class of rigid torsionfree abelian groups $A \in R\left(\mathbb{Q}^{n}\right)$. Here an abelian group $A$ is said to be rigid if its only automorphisms are the obvious ones: $a \mapsto a$ and $a \mapsto-a$. In particular, it follows that none of the isomorphism relations $\cong_{n}^{*}$ is a universal countable Borel equivalence relation. Soon afterwards, making essential use of the earlier work of Hjorth [13] and Adams-Kechris [2], Thomas [31] proved the corresponding result for the isomorphism relation $\cong_{n}$ on the class $R\left(\mathbb{Q}^{n}\right)$ of all torsion-free abelian groups of rank $n$.

Theorem 1.10 (Thomas [31]). $\left(\cong_{n}\right)<_{B}\left(\cong_{n+1}\right)$ for all $n \geq 2$.

Corollary 1.11. $\left(\cong_{n}\right)<_{B}\left(E_{\infty}\right)$ for all $n \geq 1$.

Unfortunately, while Theorem 1.10 shows that the relative complexity of the classification problem for the torsion-free abelian groups of rank $n$ increases strictly with the rank $n$, it says little about the absolute complexity of these problems. In particular, it fails to answer the following:

Question 1.12. Is the classification problem for the torsion-free abelian groups of rank 2 "genuinely difficult"? 
While it is difficult to imagine giving a precise formulation of Question 1.12, it certainly includes the question of whether $\cong_{2}$ is an immediate successor of $\cong_{1}$ with respect to Borel reducibility. In other words, does there exist a Borel equivalence relation $E$ such that

$$
\left(\cong_{1}\right)<_{B} E<_{B}\left(\cong_{2}\right) \text { ? }
$$

In seeking such an equivalence relation $E$, it is natural to consider the classification problem for various restricted classes of torsion-free abelian groups.

Definition 1.13. For each prime $p$ and $n \geq 1$, let $R^{p}\left(\mathbb{Q}^{n}\right)$ be the standard Borel space of all $p$-local subgroups $A \leqslant \mathbb{Q}^{n}$ of rank $n$ and let $\cong_{n}^{p}$ be the isomorphism relation on $R^{p}\left(\mathbb{Q}^{n}\right)$.

Here an abelian group $A$ is said to be $p$-local iff $A$ is $q$-divisible for all primes $q \neq p$. Of course, if an abelian group $A$ is $q$-divisible for all primes $q$, then $A$ is divisible and we have already seen that the divisible abelian groups are easily classified. Consequently, all of the complexity of the classification problem for the $p$-local groups is concentrated in the single prime $p$. In Thomas [31], it was shown that if the prime $p$ is fixed, then

$$
\left(\cong_{1}^{p}\right)<_{B}\left(\cong_{2}^{p}\right)<_{B} \cdots<_{B}\left(\cong_{n}^{p}\right)<_{B} \cdots .
$$

But this left open the more natural question of whether the classification problem for the $p$-local torsion-free abelian groups of a fixed rank $n \geq 2$ was strictly easier than the classification problem for arbitrary torsion-free abelian groups of rank $n$. (It is trivial that $\left(\cong_{1}^{p}\right)<_{B}\left(\cong_{1}\right)$, since there are only two $p$-local groups of rank 1 up to isomorphism; namely, $\mathbb{Q}$ and $\mathbb{Z}_{(p)}=\{a / b \in \mathbb{Q} \mid b$ is relatively prime to $p\}$.) This question was partially answered in Thomas [33], where it was shown that if $n \geq 3$ and $p \neq q$ are distinct primes, then $\cong_{n}^{p}$ and $\cong_{n}^{q}$ are incomparable with respect to Borel reducibility. Of course, this implies that if $n \geq 3$, then $\left(\cong_{n}^{p}\right)<_{B}\left(\cong_{n}\right)$ for each prime $p$. Unfortunately, the argument in Thomas [33] made essential use of the fact that if $n \geq 3$, then $\mathrm{SL}_{n}(\mathbb{Z})$ is a Kazhdan group; and, consequently, the problem remained open when $n=2$. This case was finally dealt with in Hjorth-Thomas [16], which ultimately depends upon the fact that $\mathrm{SL}_{2}(\mathbb{Z})$ satisfies a weak form of the Kazhdan property; namely, $\mathrm{SL}_{2}(\mathbb{Z})$ has Property $(\tau)$ with respect to its family of congruence subgroups. (For example, see Lubotzky [23].)

Theorem 1.14 (Hjorth-Thomas [16]). If $p \neq q$ are distinct primes, then the classification problems for the p-local and q-local torsion-free abelian groups of rank 2 are incomparable with respect to Borel reducibility.

Since it was already known [31] that $\left(\cong_{1}\right)<_{B}\left(\cong_{2}^{p}\right)$, it follows that

$$
\left(\cong_{1}\right)<_{B}\left(\cong_{2}^{p}\right)<_{B}\left(\cong_{2}\right)
$$

for each prime $p$; and hence there exists an infinite antichain of countable Borel equivalence relations which lie strictly between $\left(\cong_{1}\right)$ and $\left(\cong_{2}\right)$. With a little more effort, 
it is possible to show that there are uncountably many countable Borel equivalence relations $E$ such that

$$
\left(\cong_{1}\right)<_{B} E<_{B}\left(\cong_{2}\right) .
$$

(It should be pointed out that the proof of the following result makes essential use of the work of Kurosh-Malcev [22], [25], which was so unfairly dismissed earlier in this section.)

Definition 1.15. If $P \subseteq \mathbb{P}$ is a set of primes, then an abelian group $A$ is said to be $P$-local iff $A$ is $q$-divisible for all primes $q \notin P$.

For example, an abelian group $A$ is $\emptyset$-local iff $A$ is divisible; while, on the other hand, every abelian group is $\mathbb{P}$-local. Clearly the class of $P$-local abelian groups is included in the class of $Q$-local groups iff $P \subseteq Q$.

Theorem 1.16 (Thomas [35]). Let $n \geq 2$. If $P, Q$ are sets of primes, then the classification problem for the P-local torsion-free abelian groups of rank $n$ is Borel reducible to that for the $Q$-local groups of rank $n$ iff $P \subseteq Q$.

In particular, there exists an infinite chain $\left\{R_{m} \mid m \in \mathbb{N}\right\}$ of countable Borel equivalence relations such that

$$
\left(\cong_{1}\right)<_{B} R_{0}<_{B} R_{1}<_{B} \cdots<_{B} R_{m}<_{B} \cdots<_{B}\left(\cong_{2}\right)
$$

and so $\cong_{2}$ is very far from being an immediate successor of $\cong_{1}$ with respect to Borel reducibility.

Remark 1.17. It should be mentioned that $\mathrm{Id}_{\mathbb{R}}, E_{0}$ is the only known example (up to Borel bireducibility) of a pair of countable Borel equivalence relations $E, F$ such that $F$ is an immediate successor of $E$ with respect to $\leq_{B}$. On the other hand, there are currently no countable Borel equivalence relations $E$ with $E_{0} \leq_{B} E<_{B} E_{\infty}$ for which it is known that no such countable Borel equivalence relation $F$ exists.

\section{Superrigidity}

In this section, we shall discuss the orbit equivalence superrigidity theorems of Zimmer [38] and Furman [9], together with the corresponding Borel superrigidity theorems of Adams-Kechris [1], [2] and Thomas [32], [34]. Then, in the next section, we shall explain how to apply Borel superrigidity to the study of the classification problem for the torsion-free abelian groups of finite rank.

Recall that, by Feldman-Moore [6], if $E$ is a countable Borel equivalence relation on the standard Borel space $X$, then there exists a countable group $\Gamma$ and a Borel action of $\Gamma$ on $X$ such that $E=E_{\Gamma}^{X}$ is the corresponding orbit equivalence relation. However, it should be pointed out that the group $\Gamma$ cannot be canonically recovered from $E$; and it is usually very difficult to determine whether two given Borel actions 
of a pair $\Gamma, \Lambda$ of countable groups give rise to Borel bireducible orbit equivalence relations. Consequently, the fundamental question in the study of countable Borel equivalence relations concerns the extent to which the data $\left(X, E_{\Gamma}^{X}\right)$ determines the group $\Gamma$ and its action on $X$. In order for there to be any chance of recovering $\Gamma$ from this data, it is necessary to assume the following extra hypotheses:

(i) $\Gamma$ acts freely on $X$; i.e. $\gamma \cdot x \neq x$ for all $1 \neq \gamma \in \Gamma$ and $x \in X$.

(ii) There exists a $\Gamma$-invariant probability measure $\mu$ on $X$.

For example, by Dougherty-Jackson-Kechris [5], if (i) holds and (ii) fails, then for any countable group $\Lambda \supseteq \Gamma$, there exists a free Borel action of $\Lambda$ on $X$ such that $E_{\Lambda}^{X}=E_{\Gamma}^{X}$. If $\Gamma$ is finite, then $E_{\Gamma}^{X}$ is smooth and so we shall suppose throughout this section that $\Gamma$ is infinite. In this case, $\mu$ is necessarily nonatomic (i.e. $\mu(\{x\})=0$ for every $x \in X)$ and it follows that the probability space $(X, \mu)$ is measurably isomorphic to the unit interval $[0,1]$ equipped with its Lebesgue measure.

It is also natural to assume that the following "indecomposability hypothesis" holds:

(iii) $\Gamma$ acts ergodically on $(X, \mu)$; i.e. every $\Gamma$-invariant Borel subset of $X$ has measure 0 or 1 .

Thus, even when working in the purely Borel setting, it is useful to focus our attention on those orbit equivalence relations which arise from free ergodic actions of countable groups on probability spaces.

Example 2.1. Let $\Gamma$ be any countable group. Then the shift action of $\Gamma$ on $2^{\Gamma}$ is defined by

$$
(\gamma \cdot h)(\delta)=h\left(\gamma^{-1} \delta\right), \quad \gamma, \delta \in \Gamma, h \in 2^{\Gamma} .
$$

Let $\mu$ be the usual product probability measure on $2^{\Gamma}$. Then $\mu$ is $\Gamma$-invariant and $\Gamma$ acts ergodically on $\left(2^{\Gamma}, \mu\right)$. (For example, see Hjorth-Kechris [15].) Furthermore, letting

$$
(2)^{\Gamma}=\left\{h \in 2^{\Gamma} \mid \gamma \cdot h \neq h \text { for all } 1 \neq \gamma \in \Gamma\right\}
$$

be the free part of the action, it is easily checked that $\mu\left((2)^{\Gamma}\right)=1$.

Now suppose that $\Gamma, \Lambda$ are countable groups with free ergodic Borel actions on the probability spaces $(X, \mu),(Y, v)$ respectively. Then, by Dougherty-Jackson-Kechris [5], the corresponding countable Borel equivalence relations $E_{\Gamma}^{X}$ and $E_{\Lambda}^{Y}$ are Borel bireducible iff there exist Borel complete sections $A \subseteq X, B \subseteq Y$ such that the restricted equivalence relations are isomorphic via a Borel bijection

$$
f:\left(A, E_{\Gamma}^{X} \uparrow A\right) \cong\left(B, E_{\Lambda}^{Y} \uparrow B\right) .
$$

Here, for example, $A \subseteq X$ is said to be a complete section of $E_{\Gamma}^{X}$ iff $A$ intersects every $E_{\Gamma}^{X}$-class. In particular, it follows that $\mu(A), v(B)>0$. However, there is no 
reason to suppose that $f$ preserves the corresponding "rescaled" probability measures $\mu_{A}, v_{B}$ on $A, B$ respectively, defined by $\mu_{A}(Z)=\mu(Z) / \mu(A)$, etc. If we add the requirement that the map $f$ should also be measure-preserving, then we pass from the purely Borel setting into the richer measure-theoretic setting, where the fundamental question now concerns the extent to which the data $\left(X, E_{\Gamma}^{X}, \mu\right)$ determines the group $\Gamma$ and its action on $X$.

Definition 2.2. With the above hypotheses, the actions of $\Gamma, \Lambda$ on $(X, \mu),(Y, v)$ are said to be weakly orbit equivalent iff there exist Borel subsets $A \subseteq X, B \subseteq Y$ with $\mu(A), v(B)>0$ such that the restricted equivalence relations are isomorphic via a measure-preserving Borel bijection

$$
f:\left(A, E_{\Gamma}^{X} \uparrow A, \mu_{A}\right) \cong\left(B, E_{\Lambda}^{Y}\left\lceil B, v_{B}\right) .\right.
$$

If $\mu(A)=v(B)=1$, then the actions are said to be orbit equivalent.

Warning 2.3. At first glance, it might appear that weak orbit equivalence implies Borel bireducibility. However, this is not the case. In the measure-theoretic setting, sets and maps are only considered modulo measure zero sets; and, in particular, the Borel sets $A, B$ in Definition 2.2 are not required to be complete sections.

Definition 2.4. The actions of $\Gamma, \Lambda$ on $(X, \mu),(Y, v)$ are said to be isomorphic iff there exist

- invariant Borel subsets $X_{0} \subseteq X, Y_{0} \subseteq Y$ with $\mu\left(X_{0}\right)=v\left(Y_{0}\right)=1$,

- a measure-preserving Borel bijection $f: X_{0} \rightarrow Y_{0}$, and

- a group isomorphism $\varphi: \Gamma \rightarrow \Lambda$

such that $f(\gamma \cdot x)=\varphi(\gamma) \cdot f(x)$ for all $\gamma \in \Gamma$ and $x \in X_{0}$.

If the actions of $\Gamma, \Lambda$ on $(X, \mu),(Y, v)$ are isomorphic, then they are clearly orbit equivalent. The strongest conceivable superrigidity theorem would say that, conversely, if the actions are (weakly) orbit equivalent, then they are necessarily isomorphic. Of course, in order for anything like this to be true, it is necessary to impose strong hypotheses on the groups involved. For example, Ornstein-Weiss [27] have shown that if $\Gamma$ and $\Lambda$ are amenable groups, then any free ergodic actions of $\Gamma$, $\Lambda$ are orbit equivalent.

Definition 2.5. Let $G$ be an lcsc group and let $m$ be a fixed Haar measure on $G$. Then a subgroup $\Gamma \leqslant G$ is a lattice iff $\Gamma$ is discrete and the covolume $m(G / \Gamma)$ is finite.

Suppose now that $\Gamma$ is a lattice in a connected simple Lie group $G$ such that $\mathbb{R}$-rank $(G) \geq 2$. For example, we can take $\Gamma=\mathrm{SL}_{n}(\mathbb{Z})$ and $G=\mathrm{SL}_{n}(\mathbb{R})$ for any $n \geq 3$. Then, while the lattice $\Gamma$ is not uniquely determined by $\left(X, E_{\Gamma}^{X}, \mu\right)$, Zimmer's orbit equivalence superrigidity theorem says that this data does uniquely determine 
the ambient Lie group $G$. More precisely, suppose that $G_{0}$ and $G_{1}$ are connected centerless simple Lie groups of $\mathbb{R}$-rank at least 2 and that $\Gamma_{0}, \Gamma_{1}$ are lattices in $G_{0}, G_{1}$ respectively. (In order to keep our account as transparent as possible, we shall mainly focus on the case of lattices in connected centerless simple Lie groups.) Suppose that $\Gamma_{0}, \Gamma_{1}$ have free ergodic Borel actions on the probability spaces $\left(X_{0}, \mu_{0}\right)$ and $\left(X_{1}, \mu_{1}\right)$. Then, for each $0 \leq i \leq 1$, there is a naturally associated induced action of $G_{i}$ on the standard Borel space

$$
\widehat{X}_{i}=X_{i} \times\left(G_{i} / \Gamma_{i}\right)
$$

with invariant ergodic probability measure $\widehat{\mu}_{i}=\mu_{i} \times m_{i}$, where $m_{i}$ is the Haar probability measure on $G_{i} / \Gamma_{i}$.

Theorem 2.6 (Zimmer [38]). With the above hypotheses, if the actions of $\Gamma_{0}, \Gamma_{1}$ on $\left(X_{0}, \mu_{0}\right),\left(X_{1}, \mu_{1}\right)$ are weakly orbit equivalent, then the induced actions of $G_{0}, G_{1}$ on $\left(\widehat{X}_{0}, \widehat{\mu}_{0}\right),\left(\widehat{X}_{1}, \widehat{\mu}_{1}\right)$ are isomorphic. In particular, $G_{0} \cong G_{1}$.

Unfortunately, there are many examples of lattices $\Gamma_{0}, \Gamma_{1}$ with free ergodic Borel actions on probability spaces $\left(X_{0}, \mu_{0}\right),\left(X_{1}, \mu_{1}\right)$ for which there exists a Borel reduction $f: X_{0} \rightarrow X_{1}$ from $E_{\Gamma_{0}}^{X_{0}}$ to $E_{\Gamma_{1}}^{X_{1}}$ such that $\mu_{1}\left(f\left[X_{0}\right]\right)=0$; and hence Zimmer's orbit equivalence superrigidity theorem cannot be directly applied in the purely Borel setting. However, it was an important insight of Adams-Kechris [2] that it is possible to apply Zimmer's more fundamental cocycle superrigidity theorem. (The notion of a cocycle will not be defined in this article. Clear accounts of the theory of cocycles can be found in Zimmer [38] and Adams-Kechris [2]. In particular, Adams-Kechris [2] provides a convenient introduction to the basic techniques and results in this area, written for the non-expert in the ergodic theory of groups.)

Theorem 2.7 (Adams-Kechris [2]). With the above hypotheses, if $E_{\Gamma_{0}}^{X_{0}} \leq{ }_{B} E_{\Gamma_{1}}^{X_{1}}$, then $G_{0}$ is involved in $G_{1}$; i.e. there exist Lie subgroups $N \unlhd H \leqslant G_{1}$ such that $G_{0} \cong H / N$. Consequently, if $E_{\Gamma_{0}}^{X_{0}} \sim_{B} E_{\Gamma_{1}}^{X_{1}}$, then $G_{0} \cong G_{1}$.

Corollary 2.8 (Adams-Kechris [2]). There exist infinitely many countable Borel equivalence relations up to Borel bireducibility.

In fact, by considering Borel actions of suitable $S$-arithmetic groups for various (possibly infinite) sets of primes $S$, Adams-Kechris [2] were able to prove that there are $2^{\aleph_{0}}$ such relations up to Borel bireducibility.

Corollary 2.9 (Adams-Kechris [2]). There exist countable Borel equivalence relations which are incomparable with respect to Borel reducibility.

The methods introduced by Adams-Kechris [2] are suitable for distinguishing between orbit equivalence relations of the form $E_{\Gamma}^{X}$ and $E_{\Lambda}^{Y}$, where $\Gamma$ and $\Lambda$ are lattices in nonisogeneous higher rank semisimple Lie groups. More generally, they can be used to show that the countable Borel equivalence relations arising from suitably 
chosen actions of "large" linear groups cannot be Borel reducible to those arising from the actions of "smaller" linear groups. For example, as we shall see in the next section, a variant of Theorem 2.7 can be used to prove that if $n \geq 2$, then the orbit equivalence relation arising from the action of $\mathrm{GL}_{n+1}(\mathbb{Q})$ on the standard Borel space $R\left(\mathbb{Q}^{n+1}\right)$ of torsion-free abelian groups of rank $n+1$ is not Borel reducible to that arising from the action of $\mathrm{GL}_{n}(\mathbb{Q})$ on $R\left(\mathbb{Q}^{n}\right)$; in other words, $\left(\cong_{n+1}\right) \varliminf_{B}\left(\cong_{n}\right)$. Since we have already observed that $\left(\cong_{n}\right) \leq_{B}\left(\cong_{n+1}\right)$, this implies that $\left(\cong_{n}\right)<_{B}\left(\cong_{n+1}\right)$; i.e. that the complexity of the classification problem for the torsion-free abelian groups of rank $n$ increases strictly with the rank $n$.

However, the methods of Adams-Kechris [2] are not as well-suited for those problems which involve distinguishing between orbit equivalence relations arising from different actions of the same countable group; e.g. the isomorphism relations for the $p$-local torsion-free abelian groups of rank 2 , which arise as the orbit equivalence relations of the actions of $\mathrm{GL}_{2}(\mathbb{Q})$ on the standard Borel spaces $R^{p}\left(\mathbb{Q}^{2}\right)$. The next breakthrough occurred when Adams [1], by combining the use of Zimmer's cocycle superrigidity theorem with Ratner's measure classification theorem [29], developed a method for distinguishing between the orbit equivalence relations arising from suitably chosen actions of (not necessarily distinct) lattices $\Gamma, \Delta$ in the same higher rank semisimple Lie group $G$. (This idea had already been successfully exploited in the measure-theoretic setting by Zimmer [39] and Furman [9].) It quickly became clear that Adams' techniques were applicable to a wide range of natural problems concerning countable Borel equivalence relations. For example, combining the ideas of Adams [1] and Gefter-Golodets [10], it is straightforward to show that if $n \geq 3$, then the orbit equivalence relations arising from the following uncountable family of $\mathrm{SL}_{n}(\mathbb{Z})$-actions are pairwise incomparable with respect to Borel reducibility.

Example 2.10 (Gefter-Golodets [10]). Fix some integer $n \geq 2$ and for each nonempty set $\emptyset \neq J \subseteq \mathbb{P}$ of primes, let

$$
K_{n}(J)=\prod_{p \in J} \mathrm{SL}_{n}\left(\mathbb{Z}_{p}\right),
$$

where $\mathbb{Z}_{p}$ is the ring of $p$-adic integers. Then $K_{n}(J)$ is a compact group and we can regard $\mathrm{SL}_{n}(\mathbb{Z})$ as a subgroup of $K_{n}(J)$ via the diagonal embedding. Let $\mu_{J}$ be the Haar probability measure on $K_{n}(J)$ and let $E_{J}$ be the orbit equivalence relation arising from the free action of $\mathrm{SL}_{n}(\mathbb{Z})$ on $K_{n}(J)$ via left translations. By the Strong Approximation Theorem [28], $\mathrm{SL}_{n}(\mathbb{Z})$ is a dense subgroup of $K_{n}(J)$ and this implies that $\mathrm{SL}_{n}(\mathbb{Z})$ acts ergodically on $\left(K_{n}(J), \mu_{J}\right)$.

Theorem 2.11 (Thomas [32]). Fix some integer $n \geq 3$. If $J_{0} \neq J_{1}$ are distinct nonempty subsets of $\mathbb{P}$, then $E_{J_{0}}$ and $E_{J_{1}}$ are incomparable with respect to Borel reducibility.

The measure-theoretic analogue of this result was proved earlier by Gefter-Golodets [10], who showed that for distinct $J_{0} \neq J_{1}$, the actions of $\mathrm{SL}_{n}(\mathbb{Z})$ on $\left(K_{n}\left(J_{0}\right), \mu_{J_{0}}\right)$ 
and $\left(K_{n}\left(J_{1}\right), \mu_{J_{1}}\right)$ are not weakly orbit equivalent. More recently, Furman [9] has shown that for many free ergodic actions of lattices $\Gamma$ on probability spaces $(X, \mu)$, both the group $\Gamma$ and its action on $X$ are "almost uniquely determined" by the orbit equivalence relation $E_{\Gamma}^{X}$ and the measure $\mu$. More precisely, in our particular case, Furman's result takes the following form. (It is easily seen that if $J_{0} \neq J_{1}$, then the actions of $\mathrm{SL}_{n}(\mathbb{Z})$ on $\left(K_{n}\left(J_{0}\right), \mu_{J_{0}}\right)$ and $\left(K_{n}\left(J_{1}\right), \mu_{J_{1}}\right)$ are not virtually isomorphic. Thus the following result is strictly stronger than that of Gefter-Golodets [10].)

Theorem 2.12 (Furman [9]). Let $n \geq 3$ and let $J$ be a nonempty subset of $\mathbb{P}$. Suppose that $\Lambda$ is an arbitrary countable group with a free ergodic action on the probability space $(Y, v)$. If the actions of $\mathrm{SL}_{n}(\mathbb{Z}), \Lambda$ on the probability spaces $\left(K_{n}(J), \mu_{J}\right)$, $(Y, \nu)$ are weakly orbit equivalent, then:

(a) $\mathrm{SL}_{n}(\mathbb{Z})$ and $\Lambda$ are virtually isomorphic; and

(b) the actions of $\mathrm{SL}_{n}(\mathbb{Z}), \Lambda$ on the probability spaces $\left(K_{n}(J), \mu_{J}\right),(Y, v)$ are virtually isomorphic.

Here two countable groups $G_{0}, G_{1}$ are said to be virtually isomorphic iff there exist subgroups $H_{i} \leqslant G_{i}$ of finite index and finite normal subgroups $N_{i} \unlhd H_{i}$ for $i=0,1$ such that $H_{0} / N_{0} \cong H_{1} / N_{1}$; and the free ergodic actions of $G_{0}, G_{1}$ on the probability spaces $\left(X_{0}, \mu_{0}\right),\left(X_{1}, \mu_{1}\right)$ are said to be virtually isomorphic iff, after passing to ergodic components, the induced actions of $H_{0} / N_{0}, H_{1} / N_{1}$ on the factor spaces $\left(X_{0}, \mu_{0}\right) / N_{0},\left(X_{1}, \mu_{1}\right) / N_{1}$ are isomorphic.

No analogues of Furman's results have yet been proved in the purely Borel setting, where all of the currently known superrigidity results impose very restrictive conditions on both the domain and the range of the relevant Borel bireduction. However, it seems reasonable to conjecture that the corresponding strengthening of Theorem 2.11 also holds in this setting.

Conjecture 2.13. The conclusion of Theorem 2.12 remains true if weak orbit equivalence is replaced by Borel bireducibility.

It is not known whether the analogue of Theorem 2.11 also holds when $n=2$. Here the main obstacle is the failure of Zimmer's cocycle superrigidity theorem for the low rank Lie group $\mathrm{SL}_{2}(\mathbb{R})$. For the same reason, it is also not known whether or not these $\mathrm{SL}_{2}(\mathbb{Z})$-actions are (weakly) orbit equivalent. Since $\mathrm{SL}_{2}(\mathbb{Z})$ contains the free group $F_{2}$ on two generators as a subgroup of finite index, a positive solution of the following problem would also provide uncountably many "natural" $F_{2}$-actions which are pairwise neither Borel bireducible nor weakly orbit equivalent. Currently only three nonsmooth $F_{2}$-actions are known up to Borel bireducibility. On the other hand, in the measure-theoretic setting, Gaboriau-Popa [11] have recently constructed uncountably many $F_{2}$-actions which are pairwise not weakly orbit equivalent.

Conjecture 2.14. If $J_{0} \neq J_{1}$ are distinct nonempty sets of primes, then the actions of $\mathrm{SL}_{2}(\mathbb{Z})$ on $\left(K_{2}\left(J_{0}\right), \mu_{J_{0}}\right)$ and $\left(K_{2}\left(J_{1}\right), \mu_{J_{1}}\right)$ are neither comparable with respect to Borel bireducibility nor weakly orbit equivalent. 
We obtain a more manageable problem if we replace the lattice $\mathrm{SL}_{2}(\mathbb{Z})$ by

$$
\Gamma_{S}=\mathrm{SL}_{2}\left(\mathbb{Z}\left[1 / p_{1}, \ldots, 1 / p_{t}\right]\right)
$$

where $S=\left\{p_{1}, \ldots, p_{t}\right\}$ is a nonempty finite set of primes. Of course, $\Gamma_{S}$ is no longer a lattice in $\mathrm{SL}_{2}(\mathbb{R})$. However, if we identify $\Gamma_{S}$ with its image under the diagonal embedding into

$$
G=\mathrm{SL}_{2}(\mathbb{R}) \times \mathrm{SL}_{2}\left(\mathbb{Q}_{p_{1}}\right) \times \cdots \times \mathrm{SL}_{2}\left(\mathbb{Q}_{p_{t}}\right),
$$

then $\Gamma_{S}$ is a lattice in $G$ and Zimmer's cocycle superrigidity theorem holds for $G$. Furthermore, by Margulis-Tomanov [26], the analogue of Ratner's measure classification theorem also holds for $G$. For each nonempty (possibly infinite) set of primes $J$ such that $S \cap J=\emptyset$, let $E_{S}^{J}$ be the orbit equivalence relation arising from the action of $\Gamma_{S}$ on

$$
K_{2}(J)=\prod_{p \in J} \mathrm{SL}_{2}\left(\mathbb{Z}_{p}\right)
$$

by left translations, where $\Gamma_{S}$ is regarded as a subgroup of $K_{2}(J)$ via the diagonal embedding.

Theorem 2.15 (Thomas [34]). Suppose that $S_{0}, S_{1}$ are nonempty finite sets of primes and that $J_{0}, J_{1}$ are nonempty (possibly infinite) sets of primes such that $S_{0} \cap J_{0}=$ $S_{1} \cap J_{1}=\emptyset$. If $\left(J_{0}, S_{0}\right) \neq\left(J_{1}, S_{1}\right)$, then $E_{S_{0}}^{J_{0}}$ and $E_{S_{1}}^{J_{1}}$ are incomparable with respect to Borel reducibility.

The proof of Theorem 2.15 easily extends to the more general situation of $\Gamma_{S^{-}}$ actions on homogeneous $K_{2}(J)$-spaces. For example, it is well-known that the compact group $\mathrm{SL}_{2}\left(\mathbb{Z}_{p}\right)$ acts transitively on the projective line $\mathbb{Q}_{p} \cup\{\infty\}$ over the field of $p$-adic numbers.

Theorem 2.16 (Thomas [34]). Suppose that $p, q$ are primes and that $S, T$ are finite nonempty sets of primes such that $p \notin S, q \notin T$. If $(p, S) \neq(q, T)$, then the orbit equivalence relations of $\Gamma_{S}, \Gamma_{T}$ on the projective lines $\mathbb{Q}_{p} \cup\{\infty\}, \mathbb{Q}_{q} \cup\{\infty\}$ are incomparable with respect to Borel reducibility.

As we shall see in the next section, a variant of Theorem 2.16 can be used to prove that if $p \neq q$ are distinct primes, then the classification problems for the $p$-local and $q$-local torsion-free abelian groups of rank 2 are incomparable with respect to Borel reducibility.

\section{The classification problem for the torsion-free abelian groups of finite rank}

In this final section, we shall explain how to apply Borel superrigidity to the study of the classification problem for the torsion-free abelian groups of finite rank. First we shall 
sketch the proof of Theorem 1.10, which says that the complexity of the isomorphism relation $\cong_{n}$ for the torsion-free abelian groups of rank $n$ increases strictly with the rank $n$. This will be followed by a sketch of the proof of Theorem 1.14, which says that if $p \neq q$ are distinct primes, then the classification problems for the $p$-local and $q$-local torsion-free abelian groups of rank 2 are incomparable with respect to Borel reducibility.

Recall that for each $m \geq 1$, the isomorphism relation $\cong_{m}$ is precisely the orbit equivalence relation arising from the natural action of $\mathrm{GL}_{m}(\mathbb{Q})$ on the standard Borel space $R\left(\mathbb{Q}^{m}\right)$ of torsion-free abelian groups of rank $m$. In the last section, we saw that if $\Gamma$ is a lattice in a higher rank centerless simple Lie group $G$ and $\Gamma$ has a free ergodic action on the probability space $(X, \mu)$, then the orbit equivalence relation $E_{\Gamma}^{X}$ "encodes" the ambient Lie group $G$. More precisely, suppose that $\Lambda$ is also a lattice in a centerless simple Lie group $H$ and that $\Lambda$ has a free ergodic action on the probability space $(Y, v)$. By Theorem 2.7 , if $E_{\Gamma}^{X} \leq_{B} E_{\Lambda}^{Y}$, then $G$ is involved in $H$; and, in particular, it follows that $\operatorname{dim} G \leq \operatorname{dim} H$. This certainly suggests that the orbit equivalence relation of $\mathrm{GL}_{n+1}(\mathbb{Q})$ on $R\left(\mathbb{Q}^{n+1}\right)$ should not be Borel reducible to the orbit equivalence relation of $\mathrm{GL}_{n}(\mathbb{Q})$ on $R\left(\mathbb{Q}^{n}\right)$. Unfortunately, we cannot apply Theorem 2.7 directly to our situation, since:

(i) $\mathrm{GL}_{m}(\mathbb{Q})$ is not a lattice.

(ii) There does not exist a $\mathrm{GL}_{m}(\mathbb{Q})$-invariant probability measure on $R\left(\mathbb{Q}^{m}\right)$.

(iii) $\mathrm{GL}_{m}(\mathbb{Q})$ does not act freely on $R\left(\mathbb{Q}^{m}\right)$.

Fortunately, none of these difficulties is insurmountable. Suppose that $n \geq 2$ and that $f: R\left(\mathbb{Q}^{n+1}\right) \rightarrow R\left(\mathbb{Q}^{n}\right)$ is a Borel reduction from $\cong_{n+1}$ to $\cong_{n}$. First, following the example of Hjorth [13] and Adams-Kechris [2], we shall use the following result to deal with points (i) and (ii).

Theorem 3.1 (Hjorth [13]). For each $m \geq 2$, there exists a nonatomic $\mathrm{SL}_{m}(\mathbb{Z})$ invariant ergodic probability measure $\mu$ on $R\left(\mathbb{Q}^{m}\right)$.

In fact, Hjorth [13] has shown that for each prime $p \in \mathbb{P}$, there exists a nonatomic $\mathrm{SL}_{m}(\mathbb{Z})$-invariant ergodic probability measure $\mu_{p}$ on $R\left(\mathbb{Q}^{m}\right)$ which concentrates on the Borel subspace $R^{p}\left(\mathbb{Q}^{m}\right)$ of $p$-local groups. Later in this section, we shall sketch a proof of this result in the special case when $m=2$.

Continuing the proof of Theorem 1.10, let $E$ be the orbit equivalence relation arising from the action of the subgroup $\mathrm{SL}_{n+1}(\mathbb{Z})$ of $\mathrm{GL}_{n+1}(\mathbb{Q})$ on $R\left(\mathbb{Q}^{n+1}\right)$. Then we can regard $f$ as a countable-to-one Borel homomorphism from $E$ to $\cong_{n}$; and Theorem 1.10 is an easy consequence of the following result. (As we shall see, most of our effort during the proof of Theorem 3.3 will go into dealing with point (iii).)

Definition 3.2. If $E, F$ are equivalence relations on the standard Borel spaces $X, Y$, then the Borel map $f: X \rightarrow Y$ is a Borel homomorphism from $E$ to $F$ iff

$$
x E y \text { implies } f(x) F f(y) \text { for all } x, y \in X \text {. }
$$


Theorem 3.3 (Thomas [32]). Let $m \geq 3$ and let $X$ be a standard Borel $\mathrm{SL}_{m}(\mathbb{Z})$-space with an invariant ergodic probability measure $\mu$. Suppose that $1 \leq n<m$ and that $f: X \rightarrow R\left(\mathbb{Q}^{n}\right)$ is a Borel homomorphism from $E_{\mathrm{SL}_{m}(\mathbb{Z})}^{X}$ to $\cong_{n}$. Then there exists an $\mathrm{SL}_{m}(\mathbb{Z})$-invariant Borel subset $M$ with $\mu(M)=1$ such that $f$ maps $M$ into a single $\cong_{n}$-class.

Hence, letting $\mu$ be a nonatomic $\mathrm{SL}_{n+1}(\mathbb{Z})$-invariant ergodic probability measure on $R\left(\mathbb{Q}^{n+1}\right)$, there exists an $\mathrm{SL}_{n+1}(\mathbb{Z})$-invariant Borel subset $M \subseteq R\left(\mathbb{Q}^{n+1}\right)$ with $\mu(M)=1$ such that $f$ maps $M$ into a single $\cong_{n}$-class $\mathcal{C}$. However, this is impossible, since $f^{-1}(\mathcal{C})$ consists of only countably many $\mathrm{SL}_{n+1}(\mathbb{Z})$-orbits. Hence $\left(\cong_{n}\right)<_{B}\left(\cong_{n+1}\right)$ for all $n \geq 2$.

Next we shall sketch the proof of Theorem 3.3. Suppose that $m \geq 3$ and that $X$ is a standard Borel $\mathrm{SL}_{m}(\mathbb{Z})$-space with an invariant ergodic probability measure $\mu$. Suppose further that $1 \leq n<m$ and that $f: X \rightarrow R\left(\mathbb{Q}^{n}\right)$ is a Borel homomorphism from $E_{\mathrm{SL}_{m}(\mathbb{Z})}^{X}$ to $\cong_{n}$. We shall make use of the following variant of Theorem 2.7, which is a straightforward consequence of Zimmer's cocycle superrigidity theorem [38] and the ideas of Adams-Kechris [2].

Theorem 3.4 (Thomas [31]). Let $m \geq 3$ and let $X$ be a standard Borel $\mathrm{SL}_{m}(\mathbb{Z})$-space with an invariant ergodic probability measure $\mu$. Suppose that $H \leqslant G(\mathbb{Q})$, where $G$ is an algebraic $\mathbb{Q}$-group such that $\operatorname{dim} G<m^{2}-1$, and that $H$ acts freely on the standard Borel $H$-space $Y$. If $f: X \rightarrow Y$ is a Borel homomorphism from $E_{\mathrm{SL}_{m}(\mathbb{Z})}^{X}$ to $E_{H}^{Y}$, then there exists an $\mathrm{SL}_{m}(\mathbb{Z})$-invariant Borel subset $M \subseteq X$ with $\mu(M)=1$ such that $f$ maps $M$ into a single $H$-orbit.

As we mentioned earlier, the action of $\mathrm{GL}_{n}(\mathbb{Q})$ on $R\left(\mathbb{Q}^{n}\right)$ is not free: in fact, for each $A \in R\left(\mathbb{Q}^{n}\right)$, the stabilizer of $A$ in $\mathrm{GL}_{n}(\mathbb{Q})$ is precisely the automorphism group $\operatorname{Aut}(A)$ of $A$. Thus we are not yet in a position to apply Theorem 3.4.

Remark 3.5. This is actually a serious problem. The proof of Theorem 3.4 makes essential use of Zimmer's cocycle superrigidity theorem; and if $H$ does not act freely on $Y$, then it impossible to define the associated cocycle on which the proof depends.

From now on, let $A_{x}=f(x) \in R\left(\mathbb{Q}^{n}\right)$. Roughly speaking, our strategy will be as follows. Suppose that there exists a Borel subset $X_{0} \subseteq X$ with $\mu\left(X_{0}\right)=1$ and a fixed subgroup $L \leqslant \mathrm{GL}_{n}(\mathbb{Q})$ such that $\operatorname{Aut}\left(A_{x}\right)=L$ for all $x \in X_{0}$. Then the equivalence relation $\cong_{n} \uparrow f\left(X_{0}\right)$ will be induced by a free action of the quotient group $H=N_{\mathrm{GL}_{n}(\mathbb{Q})}(L) / L$ on the Borel subset

$$
Y=\left\{A \in R\left(\mathbb{Q}^{n}\right) \mid \operatorname{Aut}(A)=L\right\}
$$

of $R\left(\mathbb{Q}^{n}\right)$. Hence, provided that the quotient group $H$ is isomorphic to a subgroup of an algebraic $\mathbb{Q}$-group $G(\mathbb{Q})$ with $\operatorname{dim} G<m^{2}-1$, we can apply Theorem 3.4. But why should $X_{0}$ and $L$ exist? Imagine for the moment that there are only countably many possibilities for the subgroup $\operatorname{Aut}\left(A_{x}\right) \leqslant \mathrm{GL}_{n}(\mathbb{Q})$. Then there exists a Borel subset 
$Z \subseteq X$ with $\mu(Z)>0$ and a fixed subgroup $L \leqslant \mathrm{GL}_{n}(\mathbb{Q})$ such that $\operatorname{Aut}\left(A_{x}\right)=L$ for all $x \in Z$. Since $\mathrm{SL}_{m}(\mathbb{Z})$ acts ergodically on $(X, \mu)$, it follows that

$$
X_{0}=\left\{\gamma \cdot x \mid \gamma \in \operatorname{SL}_{m}(\mathbb{Z}) \text { and } x \in Z\right\} .
$$

has $\mu$-measure 1. Let $g: X \rightarrow \mathrm{SL}_{m}(\mathbb{Z})$ be a Borel function such that $g(x) \cdot x \in Z$ for all $x \in X_{0}$. Then, replacing $f$ by the Borel homomorphism $f^{\prime}$ defined by $f^{\prime}(x)=f(g(x) \cdot x)$, we can suppose that $\operatorname{Aut}\left(A_{x}\right)=L$ for all $x \in X_{0}$.

Unfortunately, this approach does not work, since there are uncountably many possibilities for the subgroup $\operatorname{Aut}\left(A_{x}\right) \leqslant \mathrm{GL}_{n}(\mathbb{Q})$. In order to get around this difficulty, we shall shift our attention from the isomorphism relation on $R\left(\mathbb{Q}^{n}\right)$ to the coarser relation of quasi-isomorphism. This relation was first introduced in Jónsson [18], where it was shown that the class of torsion-free abelian groups of finite rank has a better decomposition theory with respect to quasi-isomorphism than with respect to isomorphism. This decomposition theory will not concern us in this article. Rather we shall exploit the fact that much of the number-theoretical complexity of finite rank torsion-free abelian groups is lost when they are only considered up to quasiisomorphism; and this turns out to be enough to ensure that there are only countably many possibilities for the group of quasi-automorphisms of $A \in R\left(\mathbb{Q}^{n}\right)$.

Definition 3.6. If $A, B \in R\left(\mathbb{Q}^{n}\right)$, then $A$ and $B$ are said to be quasi-equal, written $A \approx_{n} B$, iff $A \cap B$ has finite index in both $A$ and $B$.

Definition 3.7. If $A, B \in R\left(\mathbb{Q}^{n}\right)$, then $A$ and $B$ are said to be quasi-isomorphic iff there exists $\varphi \in \mathrm{GL}_{n}(\mathbb{Q})$ such that $\varphi[A] \approx_{n} B$.

It is easily checked that $\approx_{n}$ is a countable Borel equivalence relation on $R\left(\mathbb{Q}^{n}\right)$. For each $A \in R\left(\mathbb{Q}^{n}\right)$, let $[A]$ be the $\approx_{n}$-class containing $A$. We shall consider the induced action of $\mathrm{GL}_{n}(\mathbb{Q})$ on the set of $\approx_{n}$-classes. In order to describe the setwise stabilizer in $\mathrm{GL}_{n}(\mathbb{Q})$ of a $\approx_{n}$-class $[A]$, it is first necessary to introduce the notions of a quasi-endomorphism and a quasi-automorphism. If $A \in R\left(\mathbb{Q}^{n}\right)$, then a linear transformation $\varphi \in \operatorname{Mat}_{n}(\mathbb{Q})$ is said to be a quasi-endomorphism of $A$ iff there exists an integer $m>0$ such that $m \varphi[A] \leqslant A$. In other words, $\varphi$ is a quasi-endomorphism of $A$ iff there exists an integer $m>0$ such that $m \varphi \in \operatorname{End}(A)$. It is easily checked that the collection $\mathrm{QE}(A)$ of quasi-endomorphisms of $A$ is a $\mathbb{Q}$-subalgebra of $\operatorname{Mat}_{n}(\mathbb{Q})$; and, of course, this implies that there are only countably many possibilities for $\mathrm{QE}(A)$. A linear transformation $\varphi \in \operatorname{Mat}_{n}(\mathbb{Q})$ is said to be a quasi-automorphism of $A$ iff $\varphi$ is a unit of the $\mathbb{Q}$-algebra $\mathrm{QE}(A)$; and the group of quasi-automorphisms of $A$ is denoted by $\operatorname{QAut}(A)$.

Lemma 3.8 (Thomas [31]). If $A \in R\left(\mathbb{Q}^{n}\right)$, then $\mathrm{QAut}(A)$ is the setwise stabilizer of $[A]$ in $\mathrm{GL}_{n}(\mathbb{Q})$.

In particular, there are only countably many possibilities for the setwise stabilizer of $[A]$ in $\mathrm{GL}_{n}(\mathbb{Q})$. Hence, arguing as above, we can suppose that there exists a Borel 
subset $X_{0} \subseteq X$ with $\mu\left(X_{0}\right)=1$ and a fixed subgroup $L \leqslant \mathrm{GL}_{n}(\mathbb{Q})$ such that $L$ is the setwise stabilizer of $\left[A_{x}\right]$ for all $x \in X_{0}$; and this implies that the quotient group $H=N_{\mathrm{GL}_{n}(\mathbb{Q})}(L) / L$ acts freely on the corresponding set $Y=\{[A] \mid \mathrm{QAut}(A)=L\}$ of $\approx_{n}$-classes.

Lemma 3.9 (Thomas [31]). There is an algebraic $\mathbb{Q}$-group $G$ with $\operatorname{dim} G<m^{2}-1$ such that $H \leqslant G(\mathbb{Q})$.

Consequently, we are now positioned to apply Theorem $3.4 \ldots$ except for one last complication. Unfortunately, the equivalence relation $\approx_{n}$ is not smooth and this means that $Y$ is not a standard Borel space. However, this turns out not to be a serious difficulty. As shown in Thomas [31], the equivalence relation $\approx_{n}$ is hyperfinite (which is only slightly more complicated than smooth) and Theorem 3.4 is easily extended to cover induced free actions on quotients of standard Borel spaces by hyperfinite equivalence relations.

Remark 3.10. The above argument does not go through in the case when $n=1$ because of the failure of Zimmer's cocycle superrigidity theorem for the low rank Lie group $\mathrm{SL}_{2}(\mathbb{R})$. However, as we mentioned earlier, this case had already been dealt with by Hjorth [13], who gave a completely elementary proof that $\left(\cong_{1}\right)<_{B}\left(\cong_{2}\right)$, based upon the fact that $\mathrm{GL}_{1}(\mathbb{Q})=\mathbb{Q}^{*}$ is amenable and $\mathrm{GL}_{2}(\mathbb{Q})$ is nonamenable.

In the remainder of this section, we shall sketch the proof of Theorem 1.14. This involves trying to understand the orbit equivalence relation $\cong_{2}^{p}$ of the classical group $\mathrm{GL}_{2}(\mathbb{Q})$ on the highly non-classical space $R^{p}\left(\mathbb{Q}^{2}\right)$ of $p$-local torsion-free abelian groups of rank 2. Fortunately, using the invariants of Kurosh-Malcev [22], [25], it is possible to replace $R^{p}\left(\mathbb{Q}^{2}\right)$ by a much more intelligible space.

Definition 3.11. For each prime $p$, let $E_{p}$ be the orbit equivalence relation arising from the natural action of $\mathrm{GL}_{2}(\mathbb{Q})$ on the projective line $\mathbb{Q}_{p} \cup\{\infty\}$ over the field of $p$-adic numbers.

Theorem 3.12 (Thomas [31]). $\left(\cong_{2}^{p}\right) \sim_{B}\left(E_{p}\right)$.

Thus Theorem 1.14 is an immediate consequence of the following result.

Theorem 3.13 (Hjorth-Thomas [16]). If $p \neq q$ are distinct primes, then the orbit equivalence relations $E_{p}, E_{q}$ of $\mathrm{GL}_{2}(\mathbb{Q})$ on the projective lines $\mathbb{Q}_{p} \cup\{\infty\}, \mathbb{Q}_{q} \cup\{\infty\}$ are incomparable with respect to Borel reducibility.

Sketch proof of Theorem 3.12. Following Kurosh-Malcev [22], [25], we shall describe how to assign points $V_{A} \in \mathbb{Q}_{p} \cup\{\infty\}$ to the $p$-local groups

$$
\left\{A \in R^{p}\left(\mathbb{Q}^{2}\right) \mid A \varsubsetneqq \mathbb{Q} \oplus \mathbb{Q}, \mathbb{Z}_{(p)} \oplus \mathbb{Z}_{(p)}\right\}
$$

such that:

- $A \cong B$ iff the corresponding points $V_{A}, V_{B}$ lie in the same $\mathrm{GL}_{2}(\mathbb{Q})$-orbit; 
- for each point $V \in \mathbb{Q}_{p} \cup\{\infty\}$, there exists a corresponding group $A$ such that $V_{A}=V$.

The result then follows easily from the fact that there are only countably many groups $A \in R^{p}\left(\mathbb{Q}^{2}\right)$ such that $A \cong \mathbb{Q} \oplus \mathbb{Q}, \mathbb{Z}_{(p)} \oplus \mathbb{Z}_{(p)}$.

It is first necessary to discuss the $p$-adic completion $\widehat{A}$ of each $p$-local group $A \in R^{p}\left(\mathbb{Q}^{2}\right)$, which is defined as follows. For the remainder of this proof, we shall regard $\mathbb{Q}^{2}$ as an additive subgroup of the 2-dimensional vector space $\mathbb{Q}_{p}^{2}$ over the field of $p$-adic numbers; and we shall regard $\mathrm{GL}_{2}(\mathbb{Q})$ as a subgroup of $\mathrm{GL}_{2}\left(\mathbb{Q}_{p}\right)$. For each $A \in R^{p}\left(\mathbb{Q}^{2}\right)$, let $\widehat{A}=\mathbb{Z}_{p} \otimes A$; i.e. $\widehat{A}$ is the subgroup of $\mathbb{Q}_{p}^{2}$ consisting of all finite sums

$$
\gamma_{1} a_{1}+\gamma_{2} a_{2}+\cdots+\gamma_{t} a_{t}
$$

where $\gamma_{i} \in \mathbb{Z}_{p}$ and $a_{i} \in A$ for $1 \leq i \leq t$. Then, while $A$ usually has a very complex structure, $\widehat{A}$ always decomposes into a direct sum of copies of $\mathbb{Z}_{p}$ and $\mathbb{Q}_{p}$. In fact, assuming that $A \supsetneqq \mathbb{Q} \oplus \mathbb{Q}, \mathbb{Z}_{(p)} \oplus \mathbb{Z}_{(p)}$, there exist elements $\mathbf{v}_{A}$, $\mathbf{w}_{A} \in \widehat{A}$ such that

$$
\widehat{A}=\mathbb{Q}_{p} \mathbf{v}_{A} \oplus \mathbb{Z}_{p} \mathbf{w}_{A} .
$$

(See Fuchs [8].) Let $V_{A}=\mathbb{Q}_{p} \mathbf{v}_{A}$. If $A \cong B$, then there exists $\varphi \in \mathrm{GL}_{2}(\mathbb{Q})$ such that $\varphi[A]=B$. This implies that $\varphi[\widehat{A}]=\widehat{B}$ and it follows easily that $\varphi\left[V_{A}\right]=V_{B}$. Conversely, suppose that there exists $\varphi \in \mathrm{GL}_{2}(\mathbb{Q})$ such that $\varphi\left[V_{A}\right]=V_{B}$. Since the nontrivial proper $\mathbb{Z}_{p}$-submodules of $\mathbb{Q}_{p}$ are precisely $\left\{p^{\ell} \mathbb{Z}_{p} \mid \ell \in \mathbb{Z}\right\}$, after composing $\varphi$ with a suitable transformation $v \mapsto p^{\ell} v$ if necessary, we can suppose that $\varphi[\widehat{A}]=\widehat{B}$. Since $\widehat{A} \cap \mathbb{Q}^{2}=A$ and $\widehat{B} \cap \mathbb{Q}^{2}=B$, it follows that $\varphi[A]=B$. Thus the $\mathrm{GL}_{2}(\mathbb{Q})$-orbit of the point $V_{A} \in \mathbb{Q}_{p} \cup\{\infty\}$ is a complete invariant for those $A \in R^{p}\left(\mathbb{Q}^{2}\right)$ such that $A \supsetneqq \mathbb{Q} \oplus \mathbb{Q}, \mathbb{Z}_{(p)} \oplus \mathbb{Z}_{(p)}$.

Remark 3.14. It is now easy to prove that for each prime $p \in \mathbb{P}$, there exists a nonatomic $\mathrm{SL}_{2}(\mathbb{Z})$-invariant ergodic probability measure $\mu_{p}$ on $R\left(\mathbb{Q}^{2}\right)$ which concentrates on the Borel subspace $R^{p}\left(\mathbb{Q}^{2}\right)$ of $p$-local groups. We have just seen how to assign a corresponding point $V_{A} \in \mathbb{Q}_{p} \cup\{\infty\}$ to each $A \in R^{p}\left(\mathbb{Q}^{2}\right)$ such that $A \nsubseteq \mathbb{Q} \oplus \mathbb{Q}, \mathbb{Z}_{(p)} \oplus \mathbb{Z}_{(p)}$. Conversely, for each point $V \in \mathbb{Q}_{p} \cup\{\infty\}$, there exists a corresponding group $A$ such that $V_{A}=V$. In fact, there exist countably many such groups. However, if we restrict our attention to the $\mathrm{SL}_{2}(\mathbb{Z})$-invariant Borel subset $X\left(\mathbb{Q}^{2}\right)$ consisting of those $A \in R^{p}\left(\mathbb{Q}^{2}\right)$ such that

(i) $A \supsetneqq \mathbb{Q} \oplus \mathbb{Q}, \mathbb{Z}_{(p)} \oplus \mathbb{Z}_{(p)}$,

(ii) $\mathbb{Z}_{(p)}^{2} \leqslant A$ and $\mathbb{Z}_{(p)}^{2} \nless p A$,

then we obtain a one-to-one correspondence. In summary, the map

$$
\begin{aligned}
X\left(\mathbb{Q}^{m}\right) & \rightarrow \mathbb{Q}_{p} \cup\{\infty\}, \\
A & \mapsto V_{A},
\end{aligned}
$$


is a Borel bijection satisfying $\varphi\left[V_{A}\right]=V_{\varphi[A]}$ for all $\varphi \in \mathrm{SL}_{2}(\mathbb{Z})$ and $A \in X\left(\mathbb{Q}^{2}\right)$. Hence the result follows from the observation that there exists a nonatomic $\mathrm{SL}_{2}(\mathbb{Z})$ invariant ergodic probability measure $v_{p}$ on $\mathbb{Q}_{p} \cup\{\infty\}$. To see this, recall that the compact group $K=\mathrm{SL}_{2}\left(\mathbb{Z}_{p}\right)$ acts transitively on $\mathbb{Q}_{p} \cup\{\infty\}$. Hence, letting $L$ be the stabilizer in $K$ of some point of $\mathbb{Q}_{p} \cup\{\infty\}$, we can identify the $K$-spaces $\mathbb{Q}_{p} \cup\{\infty\}$ and $K / L$. Let $v_{p}$ be the Haar probability measure on $K / L$. Since $\mathrm{SL}_{2}(\mathbb{Z})$ is a dense subgroup of $K$, it follows that $v_{p}$ is the unique $\mathrm{SL}_{2}(\mathbb{Z})$-invariant probability measure on $K / L$ and hence $\mathrm{SL}_{2}(\mathbb{Z})$ acts ergodically on $\left(K / L, v_{p}\right)$.

The above argument easily generalizes to show that for all $m \geq 2$, there exists a nonatomic $\mathrm{SL}_{m}(\mathbb{Z})$-invariant ergodic probability measure $\mu_{p}$ on $R\left(\mathbb{Q}^{m}\right)$ which concentrates on the Borel subspace consisting of those $A \in R^{p}\left(\mathbb{Q}^{m}\right)$ such that $\operatorname{dim} A / p A=1$. (For example, see Thomas [31].)

Finally we shall sketch the proof of Theorem 3.13. Recall that if $S=\left\{p_{1}, \ldots, p_{t}\right\}$ is a nonempty finite set of primes, then

$$
\Gamma_{S}=\mathrm{SL}_{2}\left(\mathbb{Z}\left[1 / p_{1}, \ldots, 1 / p_{t}\right]\right) .
$$

Also let $\Gamma_{\emptyset}=\mathrm{SL}_{2}(\mathbb{Z})$. As we shall see, Theorem 3.13 is an easy consequence of the following variant of Theorem 2.16, together with a crucial result of Hjorth [16].

Theorem 3.15 (Thomas [34]). Suppose that $p \neq q$ are distinct primes and that $S$ is a (possibly empty) finite set of primes. Let

- $E_{1}$ be the orbit equivalence relation induced by the action of $\mathrm{SL}_{2}(\mathbb{Z}[1 / q])$ on $\mathbb{Q}_{p} \cup\{\infty\}$, and

- $E_{2}$ be the orbit equivalence relation induced by the action of $\Gamma_{S}$ on $\mathbb{Q}_{q} \cup\{\infty\}$.

If $f: \mathbb{Q}_{p} \cup\{\infty\} \rightarrow \mathbb{Q}_{q} \cup\{\infty\}$ is a Borel homomorphism from $E_{1}$ to $E_{2}$, then there exists a $\mu_{p}$-measure 1 subset which is mapped to a single $E_{2}$-class.

Remark 3.16. The basic theme of Borel superigidity theory is that, under suitably strong hypotheses, every nontrivial Borel homomorphism is a "slight perturbation" of a virtual homomorphism of the corresponding measure-preserving permutation groups. In the statement of Theorem 3.15, the group $\mathrm{SL}_{2}(\mathbb{Z}[1 / q])$ was chosen because its actions on $\mathbb{Q}_{p} \cup\{\infty\}$ and $\mathbb{Q}_{q} \cup\{\infty\}$ are extremely incompatible; namely, while $\mathrm{SL}_{2}(\mathbb{Z}[1 / q])$ preserves the $p$-adic probability measure on $\mathbb{Q}_{p} \cup\{\infty\}$, there are no $\mathrm{SL}_{2}(\mathbb{Z}[1 / q])$-invariant probability measures on $\mathbb{Q}_{q} \cup\{\infty\}$.

Sketch proof of Theorem 3.13. Suppose that $f: \mathbb{Q}_{p} \cup\{\infty\} \rightarrow \mathbb{Q}_{q} \cup\{\infty\}$ is a Borel reduction between the orbit equivalence relations induced by the $\mathrm{GL}_{2}(\mathbb{Q})$-actions. Then we can regard $f$ as a countable-to-one Borel homomorphism between the $\mathrm{SL}_{2}(\mathbb{Z}[1 / q])$-action on $\mathbb{Q}_{p} \cup\{\infty\}$ and the $\mathrm{GL}_{2}(\mathbb{Q})$-action on $\mathbb{Q}_{q} \cup\{\infty\}$. Using a suitable Cocycle Reduction Theorem of Hjorth [16], we can "adjust" $f$ to obtain a 
countable-to-one Borel homomorphism $f^{\prime}: \mathbb{Q}_{p} \cup\{\infty\} \rightarrow \mathbb{Q}_{q} \cup\{\infty\}$ between the orbit equivalence relation induced by the $\mathrm{SL}_{2}(\mathbb{Z}[1 / q])$-action on $\mathbb{Q}_{p} \cup\{\infty\}$ and the orbit equivalence relation induced by the $\Gamma_{S}$-action on $\mathbb{Q}_{q} \cup\{\infty\}$ for some finite set of primes $S$, which contradicts Theorem 3.15.

In view of Remark 1.17, it would be interesting to know whether $\cong_{2}^{p}$ is an immediate successor of $\cong_{1}$ with respect to $\leq_{B}$. Equivalently:

Question 3.17. Let $E_{p}$ be the orbit equivalence relation arising from the action of $\mathrm{GL}_{2}(\mathbb{Q})$ on the projective line $\mathbb{Q}_{p} \cup\{\infty\}$ over the field of $p$-adic numbers. Does there exist a (countable) Borel equivalence relation $E$ such that $E_{0}<_{B} E<_{B} E_{p}$ ?

\section{References}

[1] Adams, S., Containment does not imply Borel reducibility. In Set Theory: The Hajnal Conference (ed. by S. Thomas), DIMACS Ser. Discrete Math. Theoret. Comput. Sci. 58, Amer. Math. Soc., Providence, RI, 2002, 1-23.

[2] Adams, S. R., Kechris, A. S., Linear algebraic groups and countable Borel equivalence relations. J. Amer. Math. Soc. 13 (2000), 909-943.

[3] Baer, R., Abelian groups without elements of finite order. Duke Math. J. 3 (1937), 68-122.

[4] Champetier, C., L'espace des groupes de type fini. Topology 39 (2000), 657-680.

[5] Dougherty, R., Jackson, S., Kechris, A. S., The structure of hyperfinite Borel equivalence relations. Trans. Amer. Math. Soc. 341 (1994), 193-225.

[6] Feldman, J., Moore, C. C., Ergodic equivalence relations, cohomology and von Neumann algebras. I, Trans. Amer. Math. Soc. 234 (1977), 289-324.

[7] Friedman, H., Stanley, L., A Borel reducibility theory for classes of countable structures. J. Symbolic Logic 54 (1989), 894-914.

[8] Fuchs, L., Infinite Abelian Groups. Vol. II, Pure and Applied Mathematics 36, Academic Press, New York, London 1973.

[9] Furman, A., Orbit equivalence rigidity. Ann. Math. 150 (1999), 1083-1108.

[10] Gefter, S. L., Golodets, V. Ya., Fundamental groups for ergodic actions and actions with unit fundamental groups. Publ. Res. Inst. Math. Sci. 24 (1988), 821-847.

[11] Gaboriau, D., Popa, S., An uncountable family of nonorbit equivalent actions of $\mathbb{F}_{n}$.J. Amer. Math. Soc. 18 (2005), 547-559.

[12] Harrington, L., Kechris, A. S., Louveau, A., A Glimm-Effros dichotomy for Borel equivalence relations. J. Amer. Math. Soc. 3 (1990), 903-927.

[13] Hjorth, G., Around nonclassifiability for countable torsion-free abelian groups. In Abelian groups and modules (Dublin, 1998), Trends Math., Birkhäuser, Basel 1999, 269-292.

[14] Hjorth, G., Kechris, A. S., Borel equivalence relations and classification of countable models. Ann. Pure Appl. Logic 82 (1996), 221-272.

[15] Hjorth, G., Kechris, A. S., Rigidity theorems for actions of product groups and countable Borel equivalence relations. Mem. Amer. Math. Soc. 177 (2005), no. 833. 
[16] Hjorth, G., Thomas, S., The classification problem for $p$-local torsion-free abelian groups of rank two. Preprint, 2004.

[17] Jackson, S., Kechris, A. S., Louveau, A., Countable Borel equivalence relations. J. Math. Logic 2 (2002), 1-80.

[18] Jónsson, B., On direct decompositions of torsion-free abelian groups. Math. Scand. 7 (1959), 361-371.

[19] Kechris, A. S., Countable sections for locally compact group actions. Ergodic Theory Dynam. Systems 12 (1992), 283-295.

[20] Kechris, A. S., Classical Descriptive Set Theory. Grad. Texts in Math. 156, Springer-Verlag, New York 1995.

[21] Kuratowski, K., Topology. Vol. I, Academic Press, New York, London 1966.

[22] Kurosh, A. G., Primitive torsionsfreie abelsche Gruppen vom endlichen Range. Ann. Math. 38 (1937), 175-203.

[23] Lubotzky, A., Discrete Groups, Expanding Graphs and Invariant Measures. Progr. Math. 125, Birkhäuser, Basel 1994.

[24] Lyndon, R. C., Schupp, P. E., Combinatorial Group Theory. Ergeb. Math. Grenzgeb. 89, Springer-Verlag, Berlin, New York 1977.

[25] Malcev, A. I., Torsion-free abelian groups of finite rank (Russian). Mat. Sb. 4 (1938), 45-68.

[26] Margulis, G. A., Tomanov, G. M., Measure rigidity for almost linear groups and its applications. J. Anal. Math. 69 (1996), 25-54.

[27] Ornstein, D., Weiss, B., Ergodic theory of amenable group actions. I. The Rohlin lemma. Bull. Amer. Math. Soc. 2 (1980), 161-164.

[28] Platonov, V., Rapinchuk, A., Algebraic Groups and Number Theory. Pure Appl. Math., Academic Press, Boston, MA, 1994.

[29] Ratner, M., On Raghunathan's measure conjecture. Ann. Math. 134 (1991), 545-607.

[30] Solovay, R. M., A model of set theory in which every set of reals is Lebesgue measurable. Ann. Math. 92 (1970), 1-56.

[31] Thomas, S., The classification problem for torsion-free abelian groups of finite rank. J. Amer. Math. Soc. 16 (2003), 233-258.

[32] Thomas, S., Superrigidity and countable Borel equivalence relations. Ann. Pure Appl. Logic 120 (2003), 237-262.

[33] Thomas, S., The classification problem for $p$-local torsion-free abelian groups of finite rank. Preprint, 2002.

[34] Thomas, S., Property $(\tau)$ and countable Borel equivalence relations. Preprint, 2004.

[35] Thomas, S., The classification problem for torsion-free abelian groups of finite rank II. In preparation.

[36] Thomas, S., Velickovic, B., On the complexity of the isomorphism relation for finitely generated groups. J. Algebra 217 (1999), 352-373.

[37] Thomas, S., Velickovic, B., On the complexity of the isomorphism relation for fields of finite transcendence degree. J. Pure Appl. Algebra. 159 (2001), 347-363.

[38] Zimmer, R. J., Ergodic Theory and Semisimple Groups. Monogr. Math. 81, Birkhäuser, Basel 1984. 
[39] Zimmer, R. J., Superrigidity, Ratner's theorem, and fundamental groups. Israel J. Math. 74 (1991), 199-207.

Mathematics Department, Rutgers University, 110 Frelinghuysen Road, Piscataway, New Jersey 08854-8019, U.S.A.

E-mail: sthomas@math.rutgers.edu 